\title{
Experimental Benchmark for Magnetic Noise and Vibrations Analysis in Electrical Machines
}

Emile Devillers, Michel Hecquet, Xavier Cimetière, Jean-Philippe Lecointe, Jean Le Besnerais, Thierry Lubin.

\begin{abstract}
This article presents an experimental setup dedicated to the analysis of magnetic noise and vibrations in radial flux electrical machines. Both electromagnetic excitation and structural response of the electrical machine are simplified to provide the first benchmark of the phenomenon of electromagnetically-excited noise and vibrations. The test bench properties as well as the magnetic, vibration and acoustic upcoming extensive measurements are shared to the scientific community, providing a clear reference case to compare different modeling and simulation approaches.
\end{abstract}

Index Terms-Electric machines, Magnetic forces, Noise, Vibrations, Experimental, Test bench.

\section{INTRODUCTION}

The vibro-acoustic performances of an electrical machine at variable-speed is a key factor in its design along electromechanical and thermal performances. Including the emitted noise and vibrations level in the design process requires a dedicated multi-physics simulation which weakly couples electromagnetic, structural mechanics and acoustic models [1], as shown in Figure 1. In particular, both electromagnetic and mechanical models have to be finely detailed to accurately compute the time and space distribution of the electromagnetic forces and the resulting vibration level of the machine's structure. Besides, for variable-speed machines such as traction machines, the simulation has to be performed for several points of the speed cycle to assert potential resonance issues between magnetic forces and structural modes of the machine [2], [3].

Therefore, this multi-physics modeling often requires a high level of discretization over time, space and speed operating points. Even if numerical models enable to accurately predict noise and vibrations levels, they are too much timeconsuming to be systematically used at early design stages [4], especially in presence of high frequency phenomenon such as Pulse Width Modulation (PWM) [5], [6]. Hence,

E.Devillers is with the L2EP laboratory, Univ. Lille, Centrale Lille, Arts et Metiers ParisTech, HEI, EA 2697, F-59000 Lille, FRANCE. He is also with the EOMYS ENGINEERING company, 59260 Hellemmes, FRANCE (email: emile.devillers@phd.centralelille.fr).

M.Hecquet is with the L2EP (email : michel.hecquet@centralelille.fr).

X.Cimetière is with the L2EP (email : xavier.cimetiere@centralelille.fr).

J-P.Lecointe is with the LSEE laboratory, Univ. Artois, EA 4025, F-62400

Bthune, FRANCE (email : jphilippe.lecointe@univ-artois.fr).

J.Le Besnerais is with EOMYS ENGINEERING (email : jean.lebesnerais@eomys.com).

T.Lubin is with the GREEN laboratory, Univ. Lorraine, EA 4366, F-54500

Vanduvre-ls-Nancy, FRANCE (email : thierry.lubin@univ-lorraine.fr).

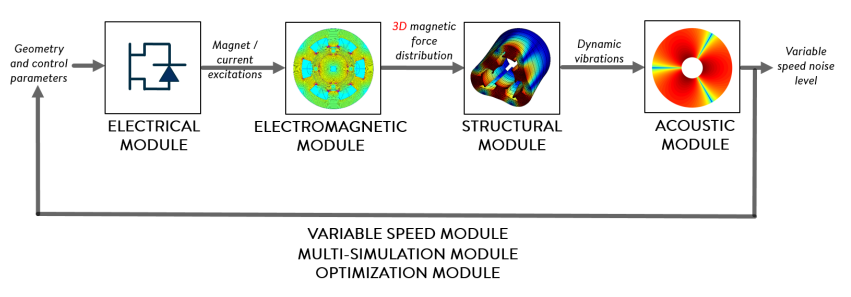

Fig. 1. Multi-physics modeling for noise and vibration assertion in electrical machines [15].

TABLE I

QUALITATIVE COMPARISON ON PERFORMANCES CRITERIA.

Tangential component

Accuracy versus computation time

Local saturation

Adaptability to complex geometries

3D Effects (skewing, end-effect)

$\begin{array}{cccc}\text { PMMF } & \text { SDM } & \text { MEC } & \text { FEM } \\ - & + & + & + \\ ++ & + & +- & -- \\ - & - & + & ++ \\ - & +- & +- & ++ \\ - & +- & +- & +\end{array}$

analytical and semi-analytical models have been developed to quickly and accurately estimate the vibro-acoustic behavior of electrical machines [7]. The main (semi-) analytical electromagnetic models are the Permeance-MagnetoMotive Force model (PMMF) [8] and the SubDomain Model (SDM) [9], followed by the Magnetic Equivalent Circuit (MEC) model. PMMF, SDM, MEC and Finite-Elements (FE) models are qualitatively compared in Table I [10].

Concerning the structural mechanics, many analytical models are applicable to predict the dynamic response of the machine's external structure. They mostly consist in representing the structure as an equivalent isotropic thin cylindrical shell or thick cylinder whose mass and stiffness have been pondered to include teeth and windings effect [1], [8], [11], [12]. The mechanical behavior of the equivalent structure is assumed to be linear. Then the dynamic response is obtained using Electromagnetic Vibration Synthesis (EVS), which consists in the superposition of the contribution of each harmonic of force pondered by its Frequency Response Function (FRF) [13]. The FRF are previously computed using analytical unit-magnitude rotating force waves [14]. The noise level is finally asserted based on the vibration velocity and the radiation factor of machine's external surface [1].

However, several key aspects of the multi-physics modeling are still under investigation in the literature, such as:

1) the interaction between radial and tangential air gap 
forces and their influence on yoke's radial vibrations [16], especially for electrical machines with few teeth and a large tooth length to yoke thickness ratio. In many analytical studies the tangential forces are neglected as their magnitude in much smaller than the one of radial forces [1], [3].

2) the spatial sampling effect of tooth on the air gap magnetic field. In case of under-sampling, force harmonics with high spatial orders may excite lower structural modes due to aliasing effect. This phenomenon exists in topologies where the number of teeth is close to the number of poles [17];

3) the computation of magnetic forces applied to a slotted structure, regarding the method formulation which depends on the electromagnetic model (mainly Virtual Work Principle for FEM [18] versus Maxwell Stress Tensor for (semi-) analytical models [1], [10]) and also on the modeling assumption (either local (or nodal) forces applied everywhere on the surface or lumped forces (resultant) applied on each tooth) whose equivalence is still under discussion [19].

4) the noise and vibrations reduction strategies including passive reduction (such as rotor and/or stator skewing [20], [21], rotor and/or stator notching [22], [23], magnetic wedges [7], flux barriers [24], etc.) and active reduction through control strategy (current injection [25], [26], effect of stator current load angle [27], damper winding [28], etc.).

Therefore, this paper deals with the design of an electrical machine prototype which aims at illustrating and answering all of the above research axes about noise and vibrations due to magnetic forces in electrical machines. This is the first part of a benchmark project on vibro-acoustics of electrical machines which wishes to improve the scientific comprehension on the topic by sharing publicly all data (including design, materials etc.) and deeply discussing the results.

As a first part of the project, this paper mainly focuses on the choice of the prototype's design to achieve the benchmark purposes. The adopted design is a Surface-mounted Permanent Magnet Synchronous Machine (SPMSM) with 12 stator slots and 5 pole pairs $(12 s 10 p)$. This choice results from several considerations on how to generate noise and vibrations in electrical machines, resulting from a pre-design based on the analytical multi-physics modeling presented in the introduction.

Then, all the prototype's characteristics are detailed including geometry and materials properties. The final design is validated with a simulation study performed on MANATEE [15], a simulation software specialized in the integrated electromagnetic and vibro-acoustic design of electrical machines. The last section describes the test bench and the different steps of the experimental benchmark, including magnetic, vibration and noise measurements.

\section{Design ApproACH AND SpecificAtions}

The main design constraint imposed to the prototype is the ability to magnetically excite several modes of the external structure in the audible frequencies. The different modes should occur at distant natural frequencies so that each resonance phenomenon can be clearly isolated and identified.

Another constraint concerns the magnetic forces production. For the sake of clarity, the prototype's design is intentionally simplified to only tackle the intrinsic complexity of magnetic forces and structural interaction. As said previously, this complexity mostly resides in the computation, projection and superposition of magnetic forces on a slotted structure including tangential and under-sampling effects (see objectives 1)-3) in the Introduction). It is easier to study these physical aspects if the harmonic content of the magnetic forces is finite, discrete, sparse and unambiguously identifiable.

Then, the slots must be sufficiently deep and large to place accelerometers on the teeth sides to observe tangential vibrations. In fact, this constraint agrees with the objective of observing tangential effects, as long teeth should emphasize the yoke displacement due to the tooth bending induced by tangential forces. Likewise, assuming large slots naturally limits the maximum number of slots hence it increases the under-sampling effect for a given number of poles.

Besides, the air gap has to be large enough to insert a network of eddy-current coil sensors and measure the time and space distribution of the air-gap flux density. To the author's knowledge, such measurement has not been carried out yet inside the air gap of electrical machines and is one of the benchmark's main original features. The influence of a large air gap on the previous constraints is mitigated. The larger is the air gap, the more spatial harmonics with high wavenumbers are filtered, but the lower are the tangential forces applied on teeth, as a direct consequence of filtering slotting harmonics.

Finally, the chosen design must comply with the assumptions of the (semi-)analytical magnetic and acoustic models presented in the introduction, in particular:

- the SubDomain Method (SDM) for electromagnetic field computation, which implies polar geometry and magnetic linearity of ferromagnetic parts ;

- acoustic analytical radiation factor including the radiation of the support frame ;

Concerning the structural modeling, investigating the interaction between magnetic forces and slotted structures is obviously contradictory with validating the structural models based on slotless equivalent cylinders. Hence the main contribution on the structural side consists in investigating and pushing the limits of the analytical structural models to estimate natural frequencies and tangential effects.

The next section explains the choice of the SPMSM $12 s 10 p$ topology based on a pre-design using the classic analytical structural regarding the previous constraints. The predesign follows a reverse engineering methodology, starting 


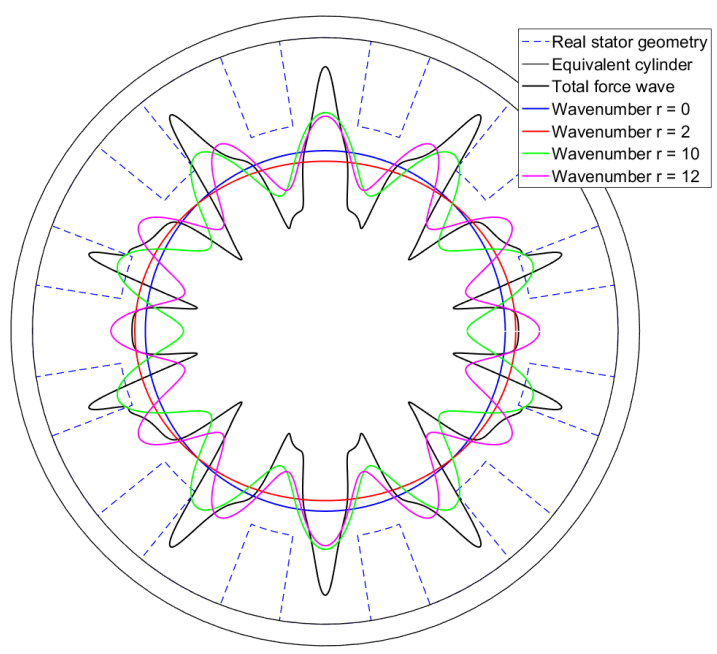

Fig. 2. Spatial harmonics of the air gap radial pressure for the adopted SPMSM $12 s 10 p$ topology at no-load and computed with SDM.

from the vibro-acoustics considerations, deducing the best electromagnetic excitation thus giving the final topology.

\section{Discussion on the Prototype Design}

\section{A. Magnetic Forces Specifications}

In this analytical pre-design, the slotted structure is transformed into an equivalent cylindrical structure. As the magnetic force is continuously applied on the interface between the air gap and the cylinder, the excitation can be equivalently seen as a force or a pressure. Furthermore, the tangential pressure is neglected as it cannot induce a radial displacement of the cylinder. The harmonic content of time and space distribution of the radial magnetic pressure $p^{r a d}(t, \theta)$ is determined by performing a 2D Fourier Transform :

$$
p^{r a d}(t, \theta)=\Re\left(\sum_{n=-\infty}^{n=+\infty} \sum_{r=-\infty}^{r=+\infty} \bar{P}_{n, r}^{r a d} e^{j(2 \pi n f t-r \theta)}\right)
$$

where:

- $\bar{P}_{n, r}^{r a d}$ is the complex magnitude of the harmonic $(n f, r)$, with the magnitude: $P_{n, r}^{r a d}=\left|\bar{P}_{n, r}^{r a d}\right|$ and the phase: $\phi_{n, r}=\arg \left(\bar{P}_{n, r}^{r a d}\right)$. As $p^{r a d}(t, \theta)$ is a real signal, $\operatorname{conj}\left(\bar{P}_{n, r}^{r a d}\right)=-\bar{P}_{-n,-r}^{r a d}$ which reduces the relevant harmonic content only to the positive frequencies.

- $(n f, r)$ is the couple of frequency and wavenumber for each harmonic. $f$ is the synchronous frequency. $n$ is a positive integer for synchronous machines and is real positive for induction machines. $r$ is a relative integer;

Fig. 2 shows the first spatial harmonics of the air gap radial pressure at a specific instant, for the adopted SPMSM topology. The real slotted geometry is represented in blue dotted line and the equivalent cylinder is the continuous external black ring. It can be seen that the main spatial harmonics of pressure are at $r=0, r=2 p=10$ and
$r=Z_{s}=12$. However, these pressure harmonics interact differently with the structure. In fact, the induced vibration level generally depends more on the frequency order and the wavenumber of the pressure harmonic than on its magnitude in case of resonance effect. The next subsection qualitatively explains the influence of the mode order and the geometrical parameters on the induced vibration level.

\section{B. Vibration Specifications}

The complex vibration level $\bar{V}_{n}^{d}$ at a given frequency derives from the dynamic displacement $\bar{U}_{n}^{d}$ of the external structure:

$$
\bar{V}_{n}^{d}=j 2 \pi n f \bar{U}_{n}^{d}
$$

The dynamic displacement $\bar{U}_{n}^{d}$ is obtained from the Electromagnetic Vibration Synthesis (EVS), which consists in summing each harmonic of pressure $\bar{P}_{n, r=m}^{r a d}$ weighted by the corresponding spatial FRF of mode order $m$ :

$$
\bar{U}_{n}^{d}=\sum_{r=-\infty}^{r=+\infty} \overline{F R F}(n f, r) \bar{P}_{n, r=m}^{r a d}
$$

The Frequency Response Function (FRF) can be actually defined as the dynamic displacement of the external structure subject to a unit-magnitude rotating force waves (meaning $\bar{P}_{n, r=m}^{r a d}=1$ ) with a certain frequency $n f$ and wavenumber $r$. The FRF can be computed using analytical models of equivalent cylinders [3], [11], finite-element models [29] or experimental modal analysis. The FRF accounts for potential resonances between each force harmonic $\bar{P}_{n, r=m}^{r a d}=1$ and structural modes of order $m$ associated to their natural frequency $f_{m}$. Under the common assumption of neglecting under-sampling effect, the resonance occurs when:

$$
r=m \quad \& \quad n f \approx f_{m}
$$

For $m \geq 2$, the classical analytical models as in [3] show that natural frequencies increase according to the mode order $m$ at the power of 2. $f_{0}$ and $f_{1}$ are usually much higher than the first natural frequencies $f_{m}$, due to the cylinder's stiffness. Natural frequencies also increase with the dimensionless geometrical ratio $R_{s y} / h_{s y}$, where $R_{s y}$ is the cylinder outer radius and $h_{s y}$ its thickness.

An analytical study also enables to show the influence on mode order and geometry parameters on the FRF. FRF maximum peak increases with the dimensionless geometrical ratio $R_{s y} / h_{s y}$ and decreases with the mode order at the power of 4 for mode orders $m \geq 2$. The structure acts like a lowpass filter on the wavenumbers, as displacements for $m=10$ and $m=12$ are much smaller than for low modes such as $m=0$ ("breathing mode") and $m=2$ ("ovalization mode"). The FRF for $m=0$ and $m=1$ have naturally their maximum peak higher than for low modes as they appear around their corresponding natural frequency.

It is worth mentioning that mode $m=1$ can be excited in presence of Unbalanced Magnetic Pull (UMP), due to 
the existence of force harmonics with wavenumber $r=1$ (induced by eccentricity, irregular magnetization, fractional winding etc.). Exciting this mode should be avoided for the prototype because it creates mechanical eccentricity and lots of parasitic forces.

As a conclusion, the prototype should generate noise and vibrations due to the excitation of low-order structural modes, in order to increase the vibration level and have the lowest values of natural frequencies. The ovalization mode (for $m=$ 2) has the lowest natural frequency and is the easiest one to be excited for the benchmark purposes. Its naturally frequency can be adjusted in the audible range by setting properly the stator dimensions. Consequently, the magnetic field should create force harmonics of wavenumber $r=2$ to excite the ovalization mode.

\section{Magnetic Field Specifications}

The aim is to produce force harmonics of wavenumber $r=2$. The magnetic force waves are created by the interaction of rotating magnetic field waves. In fact, a single rotating field wave is sufficient to create a rotating force wave and excite the structure as it is shown thereafter. The pressure distribution is given by the Maxwell Stress Tensor, such as:

$$
\begin{gathered}
p^{r a d}(t, \theta)=\frac{B^{r a d}(t, \theta)^{2}-B^{\tan }(t, \theta)^{2}}{2 \mu_{0}}\left[N . m^{-2}\right] \\
p^{\tan }(t, \theta)=\frac{B^{r a d}(t, \theta) B^{\tan }(t, \theta)}{\mu_{0}}\left[N . m^{-2}\right]
\end{gathered}
$$

where $p^{r a d}$ and $p^{\text {tan }}$ are the radial and tangential components of the magnetic pressure, $B^{r a d}$ and $B^{\text {tan }}$ are the radial and tangential components of the flux density and $\mu_{0}$ is the void magnetic permeability.

The same Fourier transform as in Eq. (1) can be done to the tangential forces and tangential flux density distributions. It is worth mentioning that both components have the same harmonic content, only the complex magnitudes differ [16]. For the numerical computation of Fourier transform, the series are truncated to a finite number of frequency and wavenumber values, respectively $N$ and $R$. Using the convolution approach, the complex magnitude of the exciting pressures is related to those of the radial and tangential flux densities as follows [30]:

$$
\begin{gathered}
\bar{P}_{n, r}^{r a d}=\frac{\bar{B}_{n, r}^{r a d} * \bar{B}_{n, r}^{r a d}-\bar{B}_{n, r}^{\tan *} \bar{B}_{n, r}^{t a n}}{2 \mu_{0}} \\
\bar{P}_{n, r}^{t a n}=\frac{\bar{B}_{n, r}^{r a d} * \bar{B}_{n, r}^{t a n}}{\mu_{0}}
\end{gathered}
$$

where $\bar{P}_{n, r}^{\tan }$ is the complex magnitude of the tangential force harmonics, $\bar{B}_{n, r}^{r a d}$ and $\bar{B}_{n, r}^{t a n}$ are the complex magnitude of the radial and tangential flux density harmonics and $*$ is the convolution operator such as:

$$
\bar{X}_{n, r} * \bar{Y}_{n, r}=\frac{1}{4 N R} \sum_{l=-N}^{l=N} \sum_{k=-R}^{k=R} \bar{X}_{l, k} * \bar{Y}_{n-l, r-k}
$$

In conclusion, due to the convolution product, a flux density harmonic either interacts with itself if $X=Y$, $l=n-l$ and $k=r-k$, or it interacts with another harmonic with different frequency order and wavenumber. A force harmonic with a low non-null wavenumber is obtained as soon as two different flux density harmonics with close wavenumbers combine themselves, even if they are flux density harmonics with high wavenumbers. This implies that the flux density spectrum should not actually be sparse to have harmonics with close wavenumbers.

Besides, force harmonics with odd wavenumbers only exist if there are both even and odd wavenumbers in the flux density spectrum. Therefore, the flux density spectrum should exclusively contain either even or odd wavenumbers to avoid exciting odd orders of structural mode, in particular $m=1$.

Finally, Eq. (9) also shows that $X$ non-null harmonics of flux density create exactly $2 X-1$ components in the pressure's spectrum. Therefore, reducing the harmonic content of the flux density reduces twice as much the one of the magnetic forces.

\section{Topology Specifications}

In real machines, the flux density's harmonics content is mainly due to the following factors:

- the discretization of the magnetic sources along the air gap (winding distribution, magnetization pattern etc.) which produces non-sinusoidal spatial MMF;

- the variation of the air gap permeance due to stator and rotor slotting effects, saturation, pole shaping etc. which modulates the MMF in time and space;

- the voltage control (such as PWM) which generates additional time harmonics in the stator current waveform.

- fault situations or manufacturing tolerances, such as demagnetization, eccentricities, broken bar etc.

Then, the sparsity of the flux density spectrum depends on the periodicity of the time and space distribution of the flux density. The spatial periodicity depends on the main topology's parameters: number of poles, phases, stator and rotor slots. The time periodicity depends on the AC supply periodicity. It also depends on if the rotor mechanical speed is synchronous or not. In induction machines, the mechanical slip generates asynchronous harmonics which coexists with synchronous ones and causes spectral leakage, except for particular values of slip. Besides, there are naturally more harmonics in induction machines than synchronous machines at variable speed due to rotor slotting and local saturation. Due to these drawbacks, induction machines are eliminated from the available topologies.

Thus the prototype is chosen among the different topologies of synchronous machines, which actually mostly differ 
on the rotor topology: Surface PM, Interior PM, Wound Rotor (WR), Synchro-Reluctant (SyRM). The main advantage of $\mathrm{PM}$ and wound rotors compared with SyRM is the ability to excite the stator only with the rotor field, hence mechanically separate the magnetic excitation from the excited structure. Besides, it does not require PWM to generate a rotating field at variable frequency, which would add many new harmonics of force. However, the rotor has to be driven by an external motor which increases the cost and the technical complexity of the test bench. Indeed, the driving machine must be vibro-acoustically isolated from the prototype to cancel any perturbations on the prototype's measurements.

The SPM rotor topology is finally retained as it is free from rotor slotting and saturation effects at no-load, which is not the case of IPM and WR.

The last step of the pre-design consists in choosing the number of pole pairs $p$ and stator slots $Z_{s}$ of the SPMSM prototype, which automatically sets the spatial periodicity of the magnetic field and forces. They must be chosen in oder to produce at least force harmonics of wavenumber $r=2$ and excite the ovalization mode. In SPMSM at no-load, the lowest non-zero wavenumber $r_{\min }$ is given by the well-known GCD rule:

$$
r_{\text {min }}=G C D\left(Z_{s}, 2 p\right)=2
$$

If $Z_{s}$ is even and $p=1, r_{\min }=\operatorname{GCD}\left(Z_{s}, 2\right)=2$ so the condition is fulfilled. However, it disagrees with the constraint of choosing a close number of poles and stator teeth, which is required to study the under-sampling effect. Besides, increasing the number of poles enables to reduce the rotating speed $N$ of the driving machine and therefore the mechanical tolerances of the test bench. The $12 s 10 p$ design is finally retained because it is a realistic topology which is also known to be particularly noisy.

With this topology, there are two harmonics of order $r=2$ at $8 f$ and $10 f$ which can excite the ovalization mode between $N=2000$ and 5000 RPM, depending on the natural frequency. However, these are not the highest excitation harmonics of order $r=2$. The highest one is the first stator slot harmonic at $\left(2 f, Z_{s}-2 p\right)$. It excites the ovalization mode if $2 f \approx f_{2}$, meaning $N=60 f / p \approx 6 f_{2}$ RPM, which requires a high speed driving machine.

\section{Test Bench Properties and Measurements}

\section{A. Setup and parameters}

The main parameters of the prototype are detailed in Fig.3 and Table II. The air gap measures $2.5 \mathrm{~mm}$ in order to setup the air gap flux density sensor. The slot is sufficient wide to stuck tri-axis accelerometers on the side of the tooth in order to measure radial and tangential vibrations at tooth tips. The lamination stack is pressurized by two lateral rings joined by four bolts. The lateral rings have holes to enable passing of instrumentation wires.

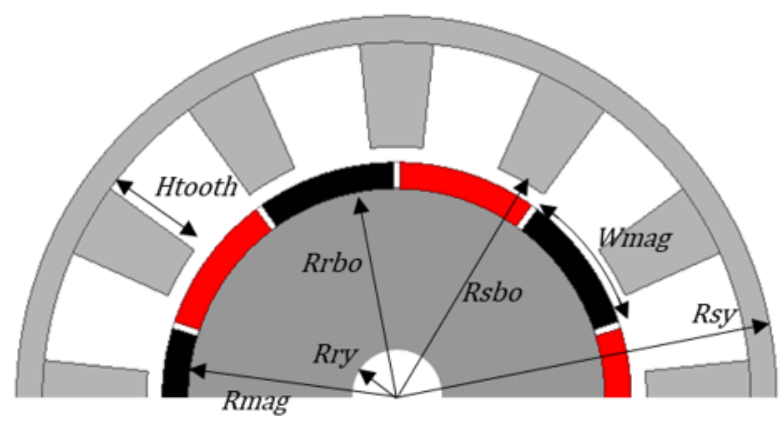

Fig. 3. Prototype SPMSM $12 s 10 p$ topology description

TABLE II

BENCHMARK PROTOTYPE PARAMETERS.

\begin{tabular}{|c|c|c|}
\hline Parameter & Symbol & Value \\
\hline Number of poles & $p$ & 10 \\
\hline Number of stator teeth & $Z_{s}$ & 12 \\
\hline Air gap length & $g$ & $2.5[\mathrm{~mm}]$ \\
\hline Stator lamination & & $M 400-50 A$ \\
\hline Stator bore radius & $R_{s b o}$ & $48[\mathrm{~mm}]$ \\
\hline Stator yoke height & $H_{s y}$ & $5[\mathrm{~mm}]$ \\
\hline Stator tooth length & $H_{\text {tooth }}$ & $20[\mathrm{~mm}]$ \\
\hline Stator outer radius & $R_{s y}$ & $73[\mathrm{~mm}]$ \\
\hline Stator stack length & $L_{s t 1}$ & $140[\mathrm{~mm}]$ \\
\hline Stator slot width & $W_{s}$ & $18[$ degrees] \\
\hline Permanent magnet & & $N d F e B(N 42)$ \\
\hline Magnet width & $W_{m a g}$ & $36[$ degrees] \\
\hline Magnet top radius & $R_{m a g}$ & $45[\mathrm{~mm}]$ \\
\hline Magnet height & $H_{m a g}$ & $5[\mathrm{~mm}]$ \\
\hline Magnet length & $L_{m a g}$ & $70[\mathrm{~mm}]$ \\
\hline Magnet sleeve height & & $0.5[\mathrm{~mm}]$ \\
\hline Magnetization orientation & & parallel [] \\
\hline Rotor steel & & $C 22(X C 18)$ \\
\hline Rotor bore radius & $R_{r b o}$ & $40[\mathrm{~mm}]$ \\
\hline Rotor shaft radius & $R_{r y}$ & $20[\mathrm{~mm}]$ \\
\hline Rotor length & $L_{s t 2}$ & $140[\mathrm{~mm}]$ \\
\hline
\end{tabular}

The test bench including the prototype and the driving machine is illustrated on Fig. 4. The driving machine is a DC machine for the easy variable speed command. Both machines are coupled with an axial flux magnetic coupling to limit the direct torque and axial force ripple transmission. They are fixed on two independent T-slot tables which are fixed on a common base frame. Silent blocks are screwed between T-slot tables and the base frame to reduce the vibration transmission through the base frame. Hence the test bench is robust to shaft eccentricity and misalignment between the two machines and the prototype is vibrationally isolated from the driving machine.

\section{B. Magnetic simulation and experimental validation}

The flux density is computed using the Subdomain method (SDM) including ferromagnetic parts with linear finite permeability [31], as shown on Fig. 5. SDM is validated with finite-element analysis and experimentation. First, the real magnetization pattern is checked by measuring the flux density on all the rotor surface using a Hall effect probe and compared with 3D FEA (not shown in this article). Then, 


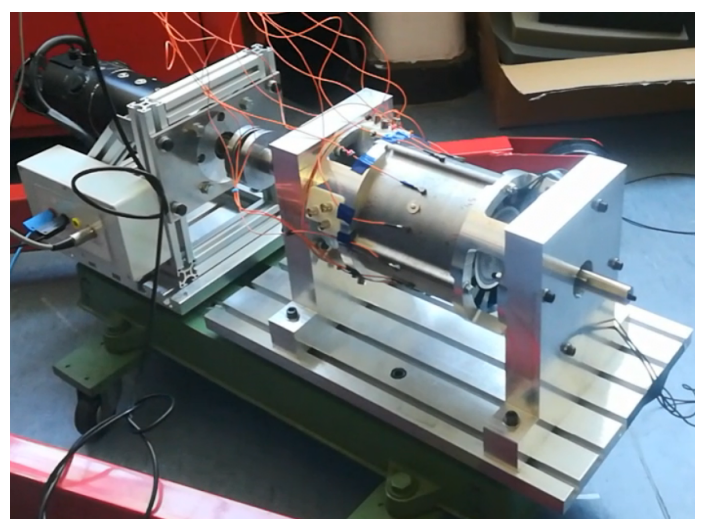

Fig. 4. Benchmark prototype (without stator) on the right and driving DC machine on the left. Twelve mono-axis accelerometers are placed on the stator surface to capture modal and operational deflection shapes.

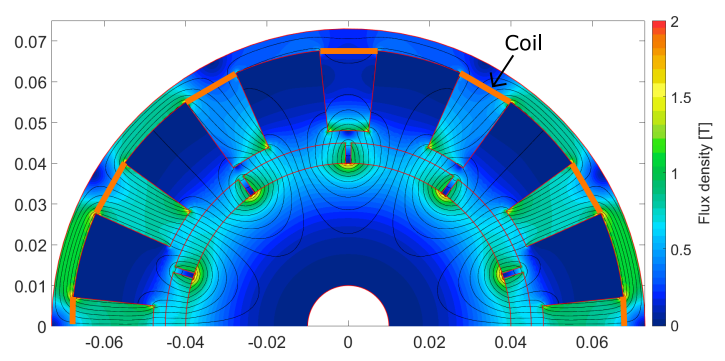

Fig. 5. Flux density and flux lines at no-load using subdomain model.

the flux density in each tooth is experimentally obtained by measuring the induced voltage in coils surrounding each tooth (represented on Fig. 5). The comparison is shown on Fig. 6 for the first tooth at $500 R P M$ and a good agreement is found between experiment and simulation.

\section{Stator modal analysis}

The stator experimental modal analysis is performed in the test bench condition. The lamination stack is pressurized by four bolts with a tightening torque of 7 N.m. The tightening torque has an effect on natural frequencies (not illustrated here) and must be known to fit with simulation models. The mode $m=0,2,3,4$ respectively appear at $f_{0}=7536 \mathrm{~Hz}$, $f_{2}=720 \mathrm{~Hz}, f_{3}=1495 \mathrm{~Hz}, f_{4}=2424 \mathrm{~Hz}$. The ovalization mode $m=2$ is easily excitable with the surface PM rotor as explained in Section III-D. The global FRF including all structural modes is illustrated on Fig. 7 and the experimental modal shapes are reconstructed on Fig. 8. Other peaks appear between $f_{4}$ and $f_{0}$ due to resonance with both circumferential and longitudinal modes.

\section{CONClusion AND Perspectives}

This article presents a test bench for the experimental study of noise and vibrations due to magnetic forces in electrical machines. The test bench aims at clearly illustrating the electromagnetic and structural interactions in electrical machines, including tangential effects and low-mode vibration generation due to teeth under-sampling effects.
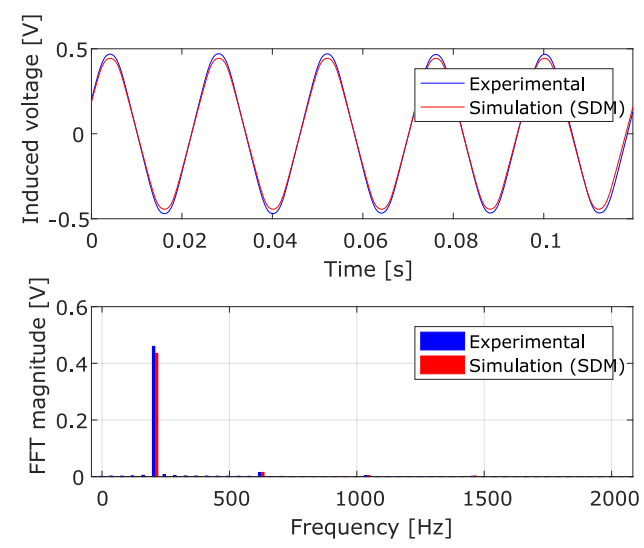

Fig. 6. Induced voltage comparison between SDM and experimentation.

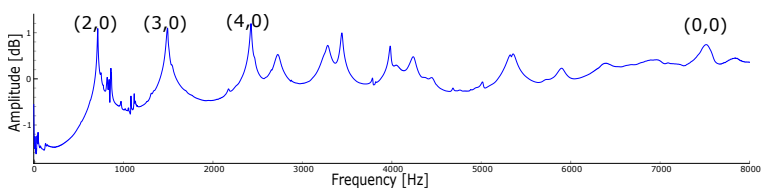

Fig. 7. Global FRF including all structural modes of the stator prototype.
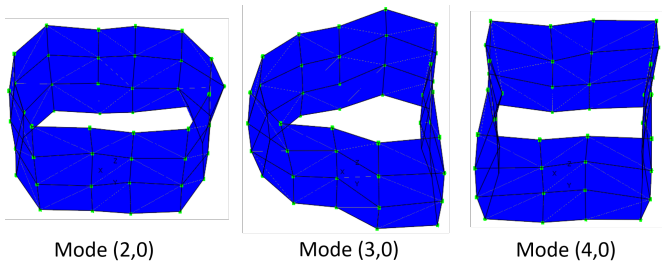

Fig. 8. Experimental modal shapes.

The second purpose is to create the first benchmark of electromagnetic and vibro-acoustic analysis in electrical machines. This benchmark consists in comparing and validating the analytical and numerical multi-physics simulations with the test bench experimental results. In perspectives, the following measurements will carried out:

- Electromagnetic air gap flux density measurement ;

- Operational modal analysis of the test bench;

- Vibration measurement including teeth radial and tangential vibrations compared with yoke vibrations;

- Acoustic and sound directivity measurement in semianechoic chamber;

- Transfer path analysis of the vibrations in the machine and its structure.

\section{REFERENCES}

[1] J. F. Gieras, C. Wang, and J. C. Lai, "Noise of polyphase electric motors," Taylor and Francis Group, p. 367, 2006.

[2] M. Boesing and R. W. De Doncker, "Exploring a Vibration Synthesis Process for the Acoustic Characterization of Electric Drives," IEEE Transactions on Industry Applications, vol. 48, no. 1, pp. 70-78, jan 2012.

[3] J. Le Besnerais, V. Lanfranchi, M. Hecquet, P. Brochet, and G. Friedrich, "Prediction of Audible Magnetic Noise Radiated by Adjustable-Speed Drive Induction Machines," IEEE Transactions on Industry Applications, vol. 46, no. 4, pp. 1367-1373, jul 2010. 
[4] P. Pellerey, V. Lanfranchi, and G. Friedrich, "Coupled Numerical Simulation Between Electromagnetic and Structural Models. Influence of the Supply Harmonics for Synchronous Machine Vibrations," IEEE Transactions on Magnetics, vol. 48, no. 2, pp. 983-986, feb 2012.

[5] J. Le Besnerais, V. Lanfranchi, M. Hecquet, and P. Brochet, "Characterization and Reduction of Audible Magnetic Noise Due to PWM Supply in Induction Machines," IEEE Transactions on Industrial Electronics, vol. 57, no. 4, pp. 1288-1295, apr 2010.

[6] M. Boesing, M. Niessen, T. Lange, and R. De Doncker, "Modeling spatial harmonics and switching frequencies in PM synchronous machines and their electromagnetic forces," in 2012 XXth International Conference on Electrical Machines. IEEE, sep 2012, pp. 3001-3007.

[7] J. Le Besnerais, "Fast prediction of variable-speed acoustic noise due to magnetic forces in electrical machines," in 2016 XXII International Conference on Electrical Machines (ICEM). Lausanne: IEEE, sep 2016, pp. 2259-2265.

[8] P. L. Timar, Noise and vibration of electrical machines. Elsevier, 1989.

[9] J. Boisson, F. Louf, J. Ojeda, X. Mininger, and M. Gabsi, "Analytical approach for magnetic and acoustic modeling of flux-switching permanent-magnet motors: Application to geometrical optimization," in 2014 17th International Conference on Electrical Machines and Systems (ICEMS), vol. 3, no. Section II. IEEE, oct 2014, pp. 30603066.

[10] E. Devillers, J. Le Besnerais, T. Lubin, M. Hecquet, and J.-P. Lecointe, "A review of subdomain modeling techniques in electrical machines: Performances and applications," in 2016 XXII International Conference on Electrical Machines (ICEM). Lausanne: IEEE, sep 2016, pp. 8692.

[11] H. Jordan, Electric motor silencer-formation and elimination of the noises in the electric motors, W.Giradet-Essen, Ed. W.Giradet-Essen, 1950.

[12] G. Verez and C. Espanet, "Natural Frequencies Analytical Modeling of Small Industrial Radial Flux Permanent Magnet Motors," 2015 18th International Conference on Electrical Machines and Systems (ICEMS), pp. 0-6, 2015.

[13] M. Boesing, "Noise and Vibration Synthesis based on Force Response Superposition,” Ph.D. dissertation, Fakultät für Elektrotechnik und Informationstechnik der Rheinisch-Westfälischen Technischen Hochschule Aachen, 2013.

[14] J. Roivainen, "Unit-wave response-based modeling of electromechanical noise and vibration of electrical machines," Ph.D. dissertation, Helsinki University of Technology, 2009.

[15] MANATEE, "Magnetic Acoustic Noise Analysis Tool for Electrical Engineering," 2018. [Online]. Available: www.manatee-software.com

[16] E. Devillers, M. Hecquet, J. Le Besnerais, and M. Regniez, "Tangential effects on magnetic vibrations and acoustic noise of induction machines using subdomain method and electromagnetic vibration synthesis," in 2017 IEEE International Electric Machines and Drives Conference (IEMDC). Miami: IEEE, may 2017, pp. 1-8.

[17] H. Fang, D. Li, R. Qu, and P. Yan, "Modulation Effect of Slotted Structure on Vibration Response in Electrical Machines," IEEE Transactions on Industrial Electronics, vol. PP, no. c, pp. 1-1, 2018.

[18] J.-1. Coulomb and G. Meunier, "Finite Element implementation of Virtual Work Principal for magnetic or electric force and torque computation," IEEE Transactions on Magnetics, vol. 20, no. 5, pp. 1894-1896, sep 1984.

[19] R. Pile, E. Devillers, and J. Le Besnerais, "Comparison of main magnetic forces computation methods for vibro-acoustic in electrical machines," IEEE Transactions on Magnetics, vol. 54, no. 7, 2018.

[20] J. Blum, J. Merwerth, and H.-G. Herzog, "Investigation of the segment order in step-skewed synchronous machines on noise and vibration," in 2014 4th International Electric Drives Production Conference (EDPC). IEEE, sep 2014, pp. 1-6.

[21] H.-Y. Yang, Y.-C. Lim, and H.-C. Kim, "Acoustic Noise/Vibration Reduction of a Single-Phase SRM Using Skewed Stator and Rotor," IEEE Transactions on Industrial Electronics, vol. 60, no. 10, pp. 42924300 , oct 2013.

[22] G.-Y. Zhou and J.-X. Shen, "Rotor Notching for Electromagnetic Noise Reduction of Induction Motors," IEEE Transactions on Industry Applications, vol. 53, no. 4, pp. 3361-3370, jul 2017.

[23] H. Lan, Q. Chen, J. Zou, Y. Xu, M. Wang, and M. Liu, "Influence of dummy slots on noise and vibration performance in permanent magnet synchronous machines," in 2017 IEEE Transportation Electrification
Conference and Expo, Asia-Pacific (ITEC Asia-Pacific). IEEE, aug 2017, pp. 1-6.

[24] G. Dajaku and D. Gerling, "A novel 12-teeth/10-poles PM machine with flux barriers in stator yoke," in 2012 XXth International Conference on Electrical Machines. IEEE, sep 2012, pp. 36-40.

[25] B. Cassoret, R. Corton, D. Roger, and J.-F. Brudny, "Magnetic noise reduction of induction machines," IEEE Transactions on Power Electronics, vol. 18, no. 2, pp. 570-579, mar 2003.

[26] D. Franck, M. Van Der Giet, K. Hameyer, M. V. D. Giet, and K. Hameyer, "Active reduction of audible noise exciting radial forcedensity waves in induction motors," 2011 IEEE International Electric Machines and Drives Conference (IEMDC), pp. 1213-1218, may 2011.

[27] W. Zhu, S. Pekarek, and B. Fahimi, "On the effect of stator excitation on radial and tangential flux and force densities in a permanent magnet synchronous machine," in IEEE International Conference on Electric Machines and Drives, 2005. IEEE, 2005, pp. 346-353.

[28] G. Bauw, F. Balavoine, B. Cassoret, O. Ninet, and R. Romary, "Damper winding for noise and vibration reduction of PWM-fed induction machines," in 2017 IEEE International Electric Machines and Drives Conference (IEMDC). IEEE, may 2017, pp. 1-6.

[29] M. Boesing, T. Schoenen, K. A. Kasper, and R. W. De Doncker, "Vibration Synthesis for Electrical Machines Based on Force Response Superposition," IEEE Transactions on Magnetics, vol. 46, no. 8, pp. 2986-2989, aug 2010.

[30] R. Rothe, M. van der Giet, and K. Hameyer, "Convolution approach for analysis of magnetic forces in electrical machines," COMPEL - The international journal for computation and mathematics in electrical and electronic engineering, vol. 29, no. 6, pp. 1542-1551, nov 2010.

[31] F. Dubas and K. Boughrara, "New Scientic Contribution on the 2-D Subdomain Technique in Polar Coordinates: Taking into Account of Iron Parts," Mathematical and Computational Applications, vol. 22, no. 1, p. 17, feb 2017.

\section{BIOGRAPHIES}

Emile Devillers is currently doing an industrial $\mathrm{PhD}$ thesis at EOMYS ENGINEERING (Lille, France) and L2EP laboratory of the Ecole Centrale de Lille, North of France. His research interests are the modeling of noise and vibrations in electrical machines due to magnetic forces for reduction purposes. He obtained a M.Sc. specialized in Energy Systems in 2015 from the Grenoble Institute of Technology, France.

Michel Hecquet received the Ph.D degree from the University of Lille, France, in 1995. His Ph.D dissertation presented a 3D permeance network of a claw-pole alternator, used for the simulation and the determination of the electromagnetic forces. Since 2008, he is full professor at Ecole Centrale de Lille in L2EP laboratory (Electrotechnic and Power Electronic Laboratory). His main interests are the development of multi-physics models of electrical machines (electromagnetic, mechanic and acoustic) and the optimal design of electrical machines.

Xavier Cimetière received the engineering degree from Ecole Centrale de Lille, Lille, France, in 1991. Since 1992, he has been a Research Engineer with the Laboratoire $\mathrm{d}^{\prime}$ Electrotechnique et $\mathrm{d}^{\prime}$ Electronique de Puissance, Ecole Centrale de Lille, Lille. His research interests include power electronics and the control of electric machines.

Jean-Philippe Lecointe, DSc, received the MSc degree in Electrical Engineering from the Universit des Sciences et Technologies de Lille, France, in 2000 . He received the $\mathrm{PhD}$ degree from the Universit dArtois, France, in 2003. He is currently Full Professor at the Artois University and director of the LSEE (Electrical Systems and Environment Research Laboratory), France. His research interests focus on electromagnetic design, efficiency, noise and vibrations of electrical machines.

Jean Le Besnerais currently works in EOMYS ENGINEERING as an R \& $\mathrm{D}$ engineer on the analysis and reduction of acoustic noise and vibrations in electrical systems. He made an industrial $\mathrm{PhD}$ thesis in Electrical Engineering at the L2EP laboratory of the Ecole Centrale de Lille, North of France, on the reduction of electromagnetic noise and vibrations in traction induction machines with ALSTOM Transport. In 2013, he founded EOMYS ENGINEERING, a company providing applied research and development services including modeling and simulation, scientific software development and experimental measurements.

Thierry Lubin received the M.Sc. degree from the University Pierre et Marie Curie, Paris 6, France in 1994 and the Ph.D. degree from the University Henri Poincar, Nancy, France, in 2003. He is currently an associate professor of electrical engineering at the University of Lorraine, at the Groupe de Recherche en Electrotechnique et Electronique de Nancy (GREEN). His interests include analytical modeling of electrical devices, contactless torque transmission systems, modeling and control of synchronous reluctance motors, and applied superconductivity in electrical engineering. 